Sarah DeMott

October 15, 2017

Collection Development Professor Michael Stoller

Major Project: Liaison Plan

\title{
MIT: Aga Khan Documentation Center (AKPDC@MIT) Liaison Plan for Visual Resource Librarian
}

The Centers provide, acquire, and borrow materials necessary to produce the best scholarship possible and through planned activities, aim to play a significant role in bringing together the various interests and fields of inquiry from both institutions. ${ }^{1}$

The Aga Khan Documentation Center at MIT is a "research center supporting teaching and scholarship on the architecture and visual cultures of Muslim societies." 2 The twofold mission is to support research through MIT/ Harvard's joint graduate program in Islamic Architecture (AKPIA@MIT); as well as encourage a wider diffusion of scholarship on Islamic art and architecture. The Aga Khan Center for Documentation is at the heart of its mission is to serve the specific needs of the MIT/Harvard community; and to make the exceptionally rich resources available to a global network of scholars. The deep quality and diversity of the collection asserts the Center's role at the forefront of scholarship on Islamic Architecture; yet strength alone is not enough to support the diverse community of users.

The MIT Aga Khan Documentation Center's community of users can be defined in three overlapping circles: MIT's School of Architecture; MIT /Harvard's Program in Islamic Architecture (AKPIA@MIT); and Aga Khan's global network of scholars on Islamic Art and Architecture. In the spirit of acknowledge AKPDC@MIT as an exemplary resource for digital scholarship as well as the Islamic world, I have designed a visualization to illustrate the network of potential connections between various actors within the AKDC@MIT’s community of users.

\footnotetext{
${ }^{1}$ https://libraries.mit.edu/akdc/collections/, October 12, 2017.

${ }^{2}$ http://libguides.mit.edu/islam-arch/? ga=2.241234433.2051331172.15080911791806775769.1505784896, October 12, 2017.
} 


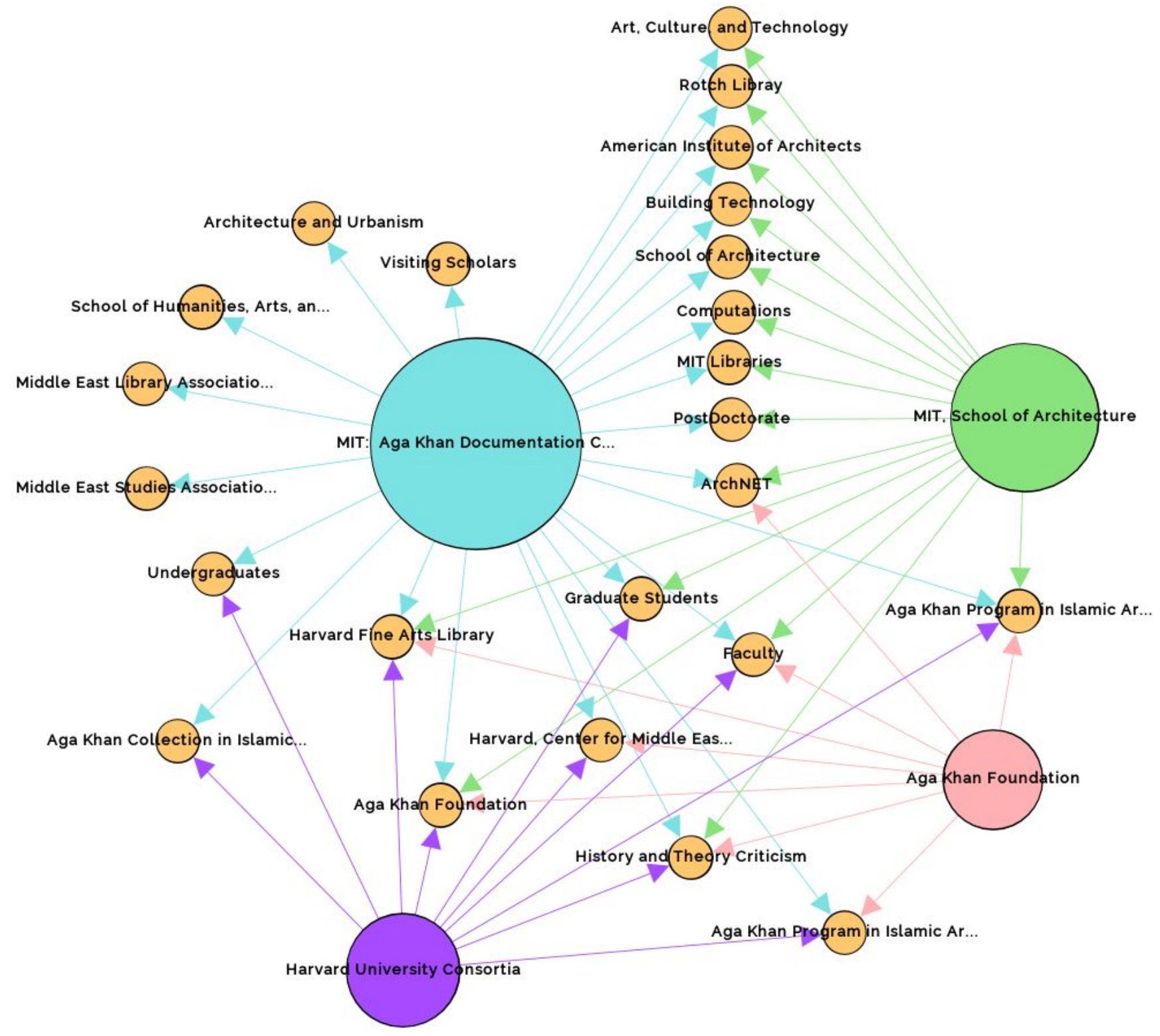

Figure 1: Connections built into the service model of liaisons.

We can see the network of actors (or community of users) through this network diagram with MIT's Aga Khan Documentation Center as the central node in blue with connections to communities of users: Green (MIT, School of Architecture): Purple (Harvard, Consortium Partnerships): and Red (Aga Khan Foundation). The network diagram illustrates the webs of overlapping liaisons that has been built into this service model.

Communication across this network is based on fundamental programmatic activities, course integration, consortium collaboration; as well as a robust state-of-the-art digital repository (ArchNET.) The AKPDC@MIT is made possible through a long-standing endowment (est. 1979) from the Agan Khan Foundation. Among the objectives of the 
Center are "planned activities" that take form in sponsored lecture series in the MIT School of Architecture, across the MIT Library's speaker's series, in collaboration with Harvard's Center for Middle East Studies (CMES.) The intensive programming and cosponsored events topics range from "Women in Art and Architecture," to "The Early Zaytuna: The Mosque of a Rebellious City," and "Conquest and Conversion in Medieval Muslim Sicily." The campus events keep the collection in the campus discourse and its materials circulating in the imagination of the academic community. The AKDC@MIT events calendar cross references calendars and list serves throughout MIT and Harvard Libraries, MIT's School of Architecture, as well as Harvard's Center for Middle East Studies (CMES.) AKPDC@MIT also publicizes its events on social media channels including a blog feed, Facebook, Twitter, and Instagram.

On campus, in addition to sponsored lectures, symposiums, conferences, and events, the AKPDC@MIT as an academic resource is also virtually present on department pages, course websites, and the Institute's LibGuides. The School of Architecture is its key subject domain, in which the Document Center serves its 56 faculty, 160 doctoral students (PhD,) 1,406 master's students (SMArchS, SMBT, SMACT,) and 45 undergraduate majors and 5 minors. Within the School of Architecture, there are five departments: History and Theory Criticism; Computations; Building Technology; Art, Culture, and Technology; and Architecture and Urbanism. Each department has a lecture series that I will plan to attend. The Aga Khan Program of Architecture (AKPIA@MIT) is within the department of History and Theory Criticism. During Fall 2017 there were four courses offered with the AKPIA@MIT, two of which (Islamic Architecture and the Environment and Orientalism and Representation) were offered by the program director Professor Naser Rabbat. The School of Architecture's website features individual biographical profiles, and is a great tool for building familiarity with students, faculty, staff and researchers. I imagine using the website as a foundation to build a database to follow the community of user's research interests and consultations. I would use the website profiles to build a bibliography of faculty publication. There is no better compliment than to be familiar with a faculty's published works. Having a knowledge of faculty research interests will put me in a position to gradually build one-on- one relationships with the 56 faculty members in the AKDC subject area. In addition to the faculty, there are two current $\mathrm{PhD}$ students in the AKAP program and I image that the Center will be a significant source of research materials for these two doctoral candidates. I would plan on providing embedded support for the four AKPIA@MIT classes each semester, the 78 graduate / 39 undergraduate courses in the architecture program, as well as suggestion to offer a workshop of research methods for the large cohort writing master thesis. I also will propose to co-teach a course in the department. I would like to acknowledge that of course, this requires that I gain the respect of the faculty and help them to see me as a resource. To that end, I've been designing a course syllabus on the subject of "Architecture and the Digital Archive." In addition to working with faculty/students in the school of architecture at MIT/Harvard, I would imagine that this course would be cross-listed with a broader interdisciplinary 
cohort in the School of Humanities, Arts, and Social Sciences and create new introductions to the AKDC@MIT collection.

The Library community is another internal resource for liaison development as the breadth of researchers interested in the collection are both specific to the AKPIA@MIT Program and the larger MIT/Harvard community of scholars. Therefore, I would be interested in meeting with Hayden Library's subject librarians in Humanities and Social Sciences, as well as colleagues (including Bibliographer in Islamic Art and Architecture and Visual Resource Librarian) at the Harvard's Fine Arts Library. I would also be eager to meet with the GIS Specialists at the Rotch Architecture Library and be sure to be well informed about their needs in terms of shape files and digital images for mapping the different historical periods and definitions of the "Islamic World'

External to the university campuses of MIT and Harvard, the AKDC also has a user constituency that is based on an affiliation with the Aga Khan Philanthropic Network. Much of the programing and networking events has a public face beyond the local community. In this public role, the staff of the AKDC represent the MIT Libraries and the Aga Khan foundation at professional associations such as Middle East Libraries Association (MELA,) Middle East Studies Association (MESA,) and American Institute of Architects. In addition to attending conferences and contributing service on national committees, the website is crucial to sustaining a global network. In 2016, AKDC was awarded "The Global Grid" for the Top 20 Architecture Websites. The on-line open access database, ArchNet is a highly innovative and user-friendly repository designed by AKDC@MIT. Off-campus, I am also interested in the opportunities to build awareness of the public architecture projects in Boston and the Middle East that the AKDC has become involved.

On an initial analysis, one might assume that the challenge for the Visual Resource Librarian for the Aga Khan Documentation Center would be maintaining the attention to detail and communication necessary to keep this highly functioning network of liaison relations in place. And one would not be wrong to assume that what is required of this position is the ability to continue coordinating this exceptional service area at a high level. In fact, the current state of AKPDC@MIT's user outreach is the very model of library liaison. Yet, as Dean Carol Mandel of NYU Libraries is keen to point out, "a fresh perspective is a remarkable thing!" Therefore, I am going to argue that the strength of the collections also has a potential to influence beyond its current audience. MIT's School of Engineering and Computer Science may be interested in working with ArchNet as a database of visual images, meta-data records, and open access platform. I ask of the AKDC if there are ways to put the material of the collection in the hands of those that are not typically working with Islamic subject material. The office of Digital 
Learning at MIT is leading the field in bold experiments in digital teaching and learning. I would look for ways to infuse the digitized subject materials found in of the Aga Khan collection into the teaching models for Digital Learning.

Finally, while asking the question of who is the user community, I wonder not only who the collection serves, but what are the effects of being housed at MIT. What is the signature strengths that make this collection thrive at MIT? What is it that MIT brings to the collaborative partnership in the subject of Islamic Art and Architecture to Harvard that is distinct to MIT's strengths and resources. More broadly, this requires liaisons to explore the nature of the MIT-Harvard partnership - what each brings to the combination. Getting to know these two immensely complex institutions is a challenge in itself.

Perhaps by defining what is MIT, while in the process of identifying who are the Aga Khan Documentation Center's users, we can develop innovative ways of enriching the collection. To start, we might note that MIT has the first department of architecture in the United States. What could this mean for the Documentation Center and its understanding of Islamic Art and Architecture in particular. We should wonder if there something about architecture that makes the study of Islam accessible? The Center has capitalized on digitizing the collections and created a cutting edge, open access, digital repository. Could we use this digital collection as a data collection as well to generate conversations about variables and structured data in the Arab world? As the Aga Khan, MIT and Harvard continue to build resources to research Islam and the Middle East, I believe the key will be to maintain its level of excellence while having one hand free to extend to new audience through multi- disciplinary uses of the source materials. 